\title{
Acknowledgement to Reviewers of Pathogens in 2018
}

\section{Pathogens Editorial Office}

MDPI, St. Alban-Anlage 66, 4052 Basel, Switzerland

Published: 8 January 2019

Rigorous peer-review is the corner-stone of high-quality academic publishing. The editorial team greatly appreciates the reviewers who contributed their knowledge and expertise to the journal's editorial process over the past 12 months. In 2018, a total of 93 papers were published in the journal, with a median time to first decision of 15 days and a median time to publication of 40 days. The editors would like to express their sincere gratitude to the following reviewers for their cooperation and dedication in 2018:

$\begin{array}{ll}\text { Abraham, Wolf-Rainer } & \text { Gupta, Kajal } \\ \text { Akio, Nakane } & \text { Hairgrove, Thomas } \\ \text { Allen, David } & \text { Halle, Stephan } \\ \text { Alsford, Sam } & \text { Hardwidge, Philip } \\ \text { Alvarez-Dominguez, Carmen } & \text { Harmon, Carrie Lapaire } \\ \text { Ambro, Lubos } & \text { Hefferon, Kathleen Laura } \\ \text { Anderson, James } & \text { Heinonen-Tanski, Helvi } \\ \text { André, Marcos Rogério } & \text { Henry, Brandon Michael } \\ \text { Ansari, Mohammad Azam } & \text { Hetman, Michal } \\ \text { Armstrong, Glen } & \text { Hill, Kent L. } \\ \text { Arthur, Arthur } & \text { Ho, Honhing } \\ \text { Attardo, Geoffrey M. } & \text { Horhat, Florin George } \\ \text { Audenaert, Kris } & \text { Hughes, Grant } \\ \text { Azab, Walid } & \text { Hummel, Mary } \\ \text { Barman, Apurba } & \text { Humphry, Roger } \\ \text { Baskakov, Ilia } & \text { Iijima, Noriaki } \\ \text { Bélard, Sabine } & \text { Ison, Michael G. } \\ \text { Beld, Joris } & \text { Jat, Parmjit S. } \\ \text { Bengtsson-Palme, Johan } & \text { Jiang, Sizun } \\ \text { Bergholz, Teresa } & \text { Johannsen, Eric } \\ \text { Berta, Graziella } & \text { Johnson, Nicholas } \\ \text { Bessa, Lucinda } & \text { Jurak, Igor } \\ \text { Bhave, Devayani } & \text { Juszczak, Lesław } \\ \text { Biegalke, Bonita } & \text { Kalsi, Megha } \\ \text { Biernasiuk, Anna } & \text { Kolton, Natalie } \\ \text { Božović, Mijat } & \text { Kradfute, Steven }\end{array}$

Peng, Teng

Perez-Gracia, Maria-Teresa

Perez-Morga, David

Petrova, Olga E.

Peyambari, Mahtab

Portilla, Maribel

Poudel, Barun

Prigigallo, Maria Isabella

Radcliff, Fiona Jane

Rather, Irfan Ahmad

Raval, Manmeet

Reichel, Michael P.

Reif, Kathryn E.

Rentsch, Doris

Roditi, Isabel

Rodrigues, Celia F.

Rodríguez-López, Pedro

Röltgen, Katharina

Romeralo, Carmen

Ronholm, Jennifer

Roze, Ludmila V.

Ruddraraju, Kasi Viswanatharaju

Russo, Annapina

Sandle, Tim

Saraiva, Margarida

Sarkar, Mrinal

Savoie, Jean-Michel

Scholte, Florine 


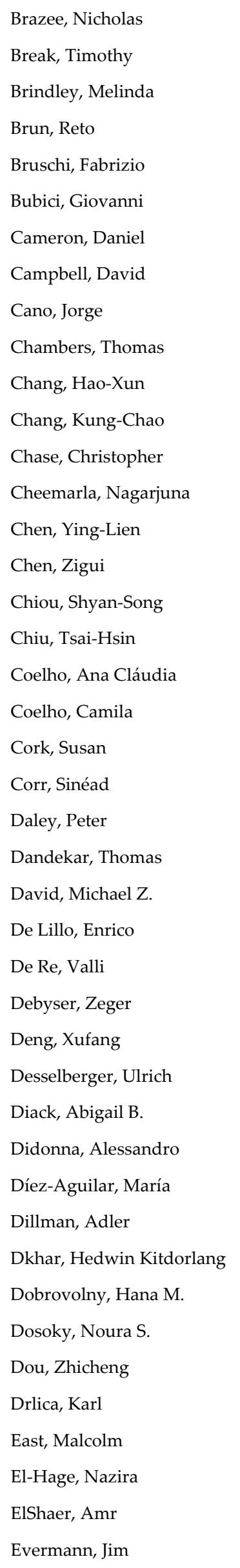

Keyhani, Nemat

Kobayashi, Nobumichi

Konstantinidis, Theocharis G.

Kozieł, Edmund

Kumar, Anil

Kumar, Binod

Kumar, Gaurav

Kumar, Rohitashw

Kumar, Sunil

Kunz, Wolfram

Kuroda, Makoto

Lahiri, Amit

Lambe, Teresa

Landolt, Gabriele A.

Lange, Dirk

LaRock, Christopher N.

Letek, Michal

Li, Shao-Gang

Li, Yan

Liang, Yuying

Lin, Xionghao

Lin, Ying-Chi

Liu, George

Liu, Jun

Łobacz, Adriana

Løbner-Olesen, Anders

Lukashevich, Igor S.

Lupien, Andréanne

Luthra, Amit

Luthra, Priya

Luvisi, Andrea

Macdonald, Andrew

Magiorkinis, Emmanouil

Manet, Evelyne

Marlicz, Wojciech

Martínez, Luis Carlos

Merda, Déborah

Metz, Stefan W.

Miki, Yasuhiro

Modhiran, Naphak

Monroy, Fernando

Montagna, Maria Teresa

Moore, Matthew D.
Selim, Sameh

Shao, Qiang

Sharma, Anurag

Sharma, Atul

Sigusch, Bernd W.

Simões, Marta Filipa

Snoeck, Chantal J.

Soultos, Nikolaos D.

Sousa, Sílvia A.

Stahelin, Robert

Stamminger, Thomas

Stefanon, Bruno

Stendahl, Olle

Sun, Xiaoming

Sunter, Jack D.

Surapathrudu, Kanakala

Suresh, Subbulakshmi

Susi, Petri

Symonds, Erin

Cornelison, Christopher T.

Tai, Wanbo

Tajima , Motoshi

Takase, Sayaka

Teixeira, Miguel C.

Tempera, Gianna

Tempera, Italo

Terzi, Valeria

Tharmalingam, Nagendran

Tiwari, Rakesh K.

Topalidou, Eleni

Toranzo, Alicia E.

Toscani, Anita

Trivedi, Pankaj

Trobaugh, Derek

Tuplin, Andrew

Upadhyay, Abhinav

Uwiera, Richard

Van Bocxlaer, Katrien

Viducic, Darija

Vigneron, Aurelien

Villani, Alessandra

Vlasova, Anastasia N.

Vylkova, Slavena 


\begin{tabular}{lll} 
Ewan, Plant & Morris, Mhairi & Walter, Monika \\
Fan, Li & Moutelikova, Romana & Wang, Zhe \\
Fan, Wei & Münz, Christian & Way, Sing Sing \\
Farrow, Mark A. & Murata, Takayuki & Weele, Pascal Van Der \\
Franz, Eelco & Musch, Mark & Wernike, Kerstin \\
Frentiu, Francesca & Naik, Surabhi & Wertz, Philip W. \\
Fusco, Vincenzina & Nevárez-Moorillón, Guadalupe Virginia & Whitehurst, Christopher \\
Futoma-Kołoch, Bożena & Niemirowicz, Gabriela T. & Wilkinson, Brian \\
García Castañeda, Javier Eduardo & Nissapatorn, Veeranoot & Willenborg, Maren \\
Garcia-Diaz, Julia & Nobbs, Angela & Wojtyczka, Robert \\
García-Díez, Juan & O'Dea, Mark A. & Wullt, Bjorn \\
Ghosh, Souvik & O'Connor, Christine & Xing, Junji \\
Gogulamudi, Venkateswara Reddy & Oh, Seung-Yoon & Yang, Tuo \\
Gold, Ben & Okino, Cintia Hiromi & Yli-Mattila, Tapani \\
Golos, Thaddeus G. & Ortiz-Urquiza, Almudena & Zampese, Enrico \\
Gonella, Elena & Osorio, Elvia Yaneth & Zhang, Fan \\
Gong, Limin & Paeshuyse, Jan & Zhang, Ling-juan \\
Goodhead, Ian & Pan, Yangang & Zhang, Ruiyong \\
Goodman, Laura & Paradkar, Prasad & Zhong, Guangming \\
Goyal, Rajni & Park, Hyun-Woo & Zhou, Bangjun \\
Gradoni, Luigi & Passler, Thomas & Zhu, Luchang \\
Guldimann, Claudia & Peng, Chen & \\
\hline & & \\
\hline & &
\end{tabular}

(C) 2019 by the author. Licensee MDPI, Basel, Switzerland. This article is an open access article distributed under the terms and conditions of the Creative Commons Attribution (CC BY) license (http://creativecommons.org/licenses/by/4.0/). 